\title{
Influence of Surface Roughness Sample Size for C-Band SAR Backscatter Applications on Agricultural Soils
}

\author{
Alex Martinez-Agirre ${ }^{\circledR}$, Jesús Álvarez-Mozos, Hans Lievens, Niko E. C. Verhoest, and Rafael Giménez
}

\begin{abstract}
Soil surface roughness determines the backscatter coefficient observed by radar sensors. The objective of this letter was to determine the surface roughness sample size required in synthetic aperture radar applications and to provide some guidelines on roughness characterization in agricultural soils for these applications. With this aim, a data set consisting of ten ENVISAT/ASAR observations acquired coinciding with soil moisture and surface roughness surveys has been processed. The analysis consisted of: 1) assessing the accuracies of roughness parameters $s$ and $l$ depending on the number of 1 -m-long profiles measured per field; 2) computing the correlation of field average roughness parameters with backscatter observations; and 3) evaluating the goodness of fit of three widely used backscatter models, i.e., integral equation model (IEM), geometrical optics model (GOM), and Oh model. The results obtained illustrate a different behavior of the two roughness parameters. A minimum of 10-15 profiles can be considered sufficient for an accurate determination of $s$, while 20 profiles might still be not enough for accurately estimating $l$. The correlation analysis revealed a clear sensitivity of backscatter to surface roughness. For sample sizes $>15$ profiles, $R$ values were as high as 0.6 for $s$ and $\sim 0.35$ for $l$, while for smaller sample sizes $R$ values dropped significantly. Similar results were obtained when applying the backscatter models, with enhanced model precision for larger sample sizes. However, IEM and GOM results were poorer than those obtained with the Oh model and more affected by lower sample sizes, probably due to larger uncertainly of $l$.
\end{abstract}

Index Terms-Agricultural soils, backscatter models, surface roughness, synthetic aperture radar (SAR).

\section{INTRODUCTION}

S YNTHETIC aperture radar (SAR) sensors measure the backscatter of observed targets and offer valuable information for the identification of terrain covers and for the

Manuscript received August 31, 2017; accepted October 8, 2017. This work was supported by the Spanish Ministry of Economy and Competitiveness through scholarship under Grant BES-2012-054521 and through MINECO/FEDER, EU, under Project CGL2011-24336, Project CGL201564284-C2-1-R, and Project CGL2016-75217-R. (Corresponding author: Alex Martinez-Agirre.)

A. Martinez-Agirre, J. Álvarez-Mozos, and R. Giménez are with the Department of Projects and Rural Engineering, IS-FOOD Institute (Innovation and Sustainable Development in Food Chain), Public University of Navarre, 31006 Pamplona, Spain (e-mail: alejandro.mda@unavarra.es).

$\mathrm{H}$. Lievens is with the Laboratory of Hidrology and Water Management, Ghent University, B-9000 Ghent, Belgium, and also with the Global Modeling and Assimilation Office, NASA Goddard Space Flight Center, Greenbelt, MD 20771 USA.

N. E. C. Verhoest is with the Laboratory of Hidrology and Water Management, Ghent University, B-9000 Ghent, Belgium.

Color versions of one or more of the figures in this letter are available online at http://ieeexplore.ieee.org.

Digital Object Identifier 10.1109/LGRS.2017.2762434 retrieval of biogeophysical parameters of interest, such as soil moisture (SM), vegetation phenology, and biomass. Among other terrain parameters, soil surface roughness (SSR) strongly affects the scattering of microwaves, and hence largely determines the backscatter coefficient $\left(\sigma^{0}\right)$ observed by radar sensors, complicating the interpretation and analysis of SAR data [1]. In the SAR literature, SSR has been mostly parameterized by the standard deviation of the heights $(s)$, the correlation length $(l)$, and the shape of the autocorrelation function [2], generally assumed exponential for agricultural soils. Several backscatter models exist that use these parameters as input for simulating $\sigma^{0}$. If backscatter observations are available, models can be inverted for retrieving a certain terrain parameter of interest (normally SM). An accurate estimation of roughness parameters is a prerequisite for this. Yet, their spatial variability and also the multiscalar nature of roughness make it difficult to determine $s$ and $l$ values with the required accuracy for obtaining useful inversions [2].

Surface roughness is known to be a multiscalar phenomenon, causing instruments with different measuring ranges (i.e., profile length or surveying area) yield parameter values that are not comparable with each other [2]. In particular, the presence of long-wavelength roughness components (i.e., several meters) on a soil surface or profile can strongly affect the shape of the obtained autocorrelation functions, introducing uncertainty in the determination of $l$ [3]. On the other hand, recent research has evidenced that these long-wavelength components might not play a significant role in the scattering of microwaves at the frequencies used by earth observation satellites [4], [5]. This is in line with previous studies that used profile lengths of 1-2 $\mathrm{m}$ for surface roughness characterization with good results [6], [7].

However, due to the spatial variability of surface roughness, a minimum amount of samples might be required for accurately characterizing roughness parameters for a particular agricultural field or roughness class. Bryant et al. [8] observed that at least 20 profiles were required to accurately determine $s$. Similarly, Baghdadi et al. [9] reported a $\pm 10 \%$ accuracy for parameter $s$ and $\pm 20 \%$ for $l$ when ten roughness profiles were used. Yet, it is necessary not only to assess how the roughness sample size (i.e., number of profiles measured) affects the accuracy of the computed parameters, but also to evaluate how it influences the accuracy of backscatter simulations using observed $\sigma^{0}$ data.

The aim of this letter was to evaluate the influence of surface roughness sample size on SAR backscattering in different 
TABLE I

Roughness Classes CorResponding to EACH Field AND Measurement Date. Four 5-m-Long Roughness PROFILES WERE ACOUIRED PER FIELD

\begin{tabular}{ccccc}
\hline Field ID. & $\mathbf{2 2 / 0 9 / 2 0 0 4}$ & $\mathbf{0 8 / 1 0 / 2 0 0 4}$ & $\mathbf{2 4 / 1 0 / 2 0 0 4}$ & $\mathbf{1 7 / 1 2 / 2 0 0 4}$ \\
\hline 188 & HR & HR & - & $\mathrm{P}$ \\
189 & HR & HR & HS & P \\
193 & HR & HR & P & P \\
194 & - & HR & HR & P \\
199 & MP & MP & MP & P \\
201 & HS & HS & - & P \\
208 & MP & - & - & PC \\
235 & HS & HS & P & P \\
255 & HS & HS & - & P \\
258 & HR & - & - & P \\
\hline
\end{tabular}

- Fields not monitored on that particular day

agricultural soils. The objective was to determine the minimum number of 1-m-long profiles required in SAR applications and to provide same guidelines on how roughness should be characterized for these applications. With this aim, a data set consisting of ENVISAT/ASAR observations acquired coinciding with some ground surveys has been processed. The analysis consisted of: 1) assessing the accuracies of $s$ and $l$ depending on the number of profiles measured per field; 2) computing the correlation of field average roughness parameters with backscatter; and 3) evaluating the goodness of fit of backscatter models depending on the roughness sample size considered.

\section{Materials And Methods}

\section{A. Test Site}

The experimental data acquisition was carried out on the watershed of La Tejería (N42 $44^{\prime} 10.6^{\prime \prime}$ and W1 $\left.{ }^{\circ} 56^{\prime} 57.2^{\prime \prime}\right)$ in Navarre (Spain) [10]. The climate is humid sub-Mediterranean with a mean annual temperature of $13{ }^{\circ} \mathrm{C}$ and an average annual precipitation of $\sim 700 \mathrm{~mm}$. Soils have a silty-clay texture and are relatively shallow (0.5-1 m deep). Ten agricultural fields were studied with an area ranging between 3 and 7.3 ha.

Soil preparation operations were performed sequentially during September and October 2004 for cultivating winter cereal. Five different tillage treatments were observed from September to December 2004 (Table I): mouldboard plough (MP), harrowed rough (HR), harrowed smooth (HS), planted (P), and planted compacted (PC).

\section{B. Surface Roughness Data}

Surface roughness was measured using a 5-m-long laser profile meter with a resolution of $5 \mathrm{~mm}$ and a vertical accuracy of $1.25 \mathrm{~mm}$ [11]. On each monitored field, four 5-m-long profiles were measured per date (except for field 208 on September 22, 2004), spatially distributed throughout the field and in parallel to the tillage direction. Each acquired profile was subdivided into five 1-m-long profiles, and these were detrended using a linear function to subtract the terrain slope. Thus, 20 1-m-long profiles (i.e., independent samples) were obtained per field, making a total of 6351 -m-long profiles.
Two standard surface roughness parameters were analyzed: the standard deviation of surface heights $(s)$ and the correlation length $(l)$ obtained considering an exponential autocorrelation function [2]. Further details on the processing of profiles and roughness parameters are available in [11].

\section{Soil Moisture Data}

SM was measured using a commercial time domain reflectometry (TDR) instrument. On each field, five spatially distributed measurement locations were monitored per date. Soil samples were used to calibrate the TDR probe. Also, TOPLATS [12]-modeled SM values were used for four satellite acquisition dates (Table II) on which the TDR measurements were not available.

\section{SAR Data}

Ten ENVISAT/ASAR scenes were acquired over La Tejería watershed during the study period (Table II). Scenes were acquired as VV single-pol image mode precision image products in swath IS2 (except for September 22, 2004, that was VV/HH Alternate Pol in IS1), half of them in ascending pass and the other half in descending. In all cases, the resolution was $30 \mathrm{~m} \times 30 \mathrm{~m}$. Scenes were: 1) orthorectified (with an error $<1$ pixel); 2) calibrated (using the local incidence angle); and 3 ) speckle filtered (gamma MAP filter with a window of $5 \times 5$ ). Mean backscatter coefficient values $\sigma^{0}$ were calculated for each field per date.

\section{E. Data Analysis}

The analysis presented here focused on the influence of sample size on the characterization of surface roughness for SAR applications. For this, an increasing number of 1-m-long roughness profiles (from 1 to 20) were considered for each field, and the following analyses were carried out: 1) assessment of the behavior of roughness parameters; 2) evaluation of the correlation between normalized $\sigma^{0}$ and roughness parameters; and 3) evaluation of the goodness of fit of different backscatter models.

The behavior of roughness parameters was evaluated by comparing the average and standard deviation of $s$ and $l$ per class computed considering an increasing sample size (i.e., number of profiles). For the correlation analysis, field average $\sigma^{0}$ values were normalized for incidence angle and SM variations, so as to remove the influence of factors other than surface roughness on $\sigma^{0}$ values [5]. Further details on the normalization can be found in [5]. The Spearman coefficient $(R)$ was computed between the $\sigma_{\text {norm }}^{0}$ (normalized $\sigma^{0}$ ) series and field average $s$ and $l$ values considering an increasing sample size. Finally, the goodness of fit of three backscatter models was evaluated by computing the root-mean-square error (RMSE) between observed $\sigma^{0}$ values and simulated ones; the latter were obtained using field average $s$ and $l$ values for an increasing sample size. Due to their different nature and validity range, three backscatter models were considered: the physically based integral equation model (IEM) [13] and geometrical optics model (GOM) [2] for the smooth (P and PC) 
TABLE II

SUMMARY OF SAR DATA

\begin{tabular}{clccccc}
\hline Date & SAR data & $\boldsymbol{\theta}_{\text {LOC }}\left(^{\circ}\right)^{*}$ & Pass & No. Fields & Roughness data & SM data \\
\hline $22 / 09 / 2004$ & Envisat/ASAR & $7.2-16.2$ & Descending & 9 & Profilometer & TDR \\
$08 / 10 / 2004$ & Envisat/ASAR & $11.6-20.9$ & Descending & 8 & Profilometer & TDR \\
$11 / 10 / 2004$ & Envisat/ASAR & $20.9-31.4$ & Ascending & 8 & $=$ & TOPLATS \\
$24 / 10 / 2004$ & Envisat/ASAR & $15.7-24.9$ & Descending & 5 & Profilometer & TDR \\
$27 / 10 / 2004$ & Envisat/ASAR & $16.9-27.2$ & Ascending & 5 & $=$ & TOPLATS \\
$17 / 12 / 2004$ & Envisat/ASAR & $11.6-20.9$ & Descending & 10 & Profilometer & TDR \\
$20 / 12 / 2004$ & Envisat/ASAR & $20.8-31.2$ & Ascending & 10 & $=$ & TOPLATS \\
$02 / 01 / 2005$ & Envisat/ASAR & $15.8-24.5$ & Descending & 10 & $=$ & TDR \\
$05 / 01 / 2005$ & Envisat/ASAR & $16.8-26.9$ & Ascending & 10 & $=$ & TDR \\
$24 / 01 / 2005$ & Envisat/ASAR & $20.9-31.3$ & Ascending & 10 & $=$ & TOPLATS \\
\hline
\end{tabular}

*Local incidence angle
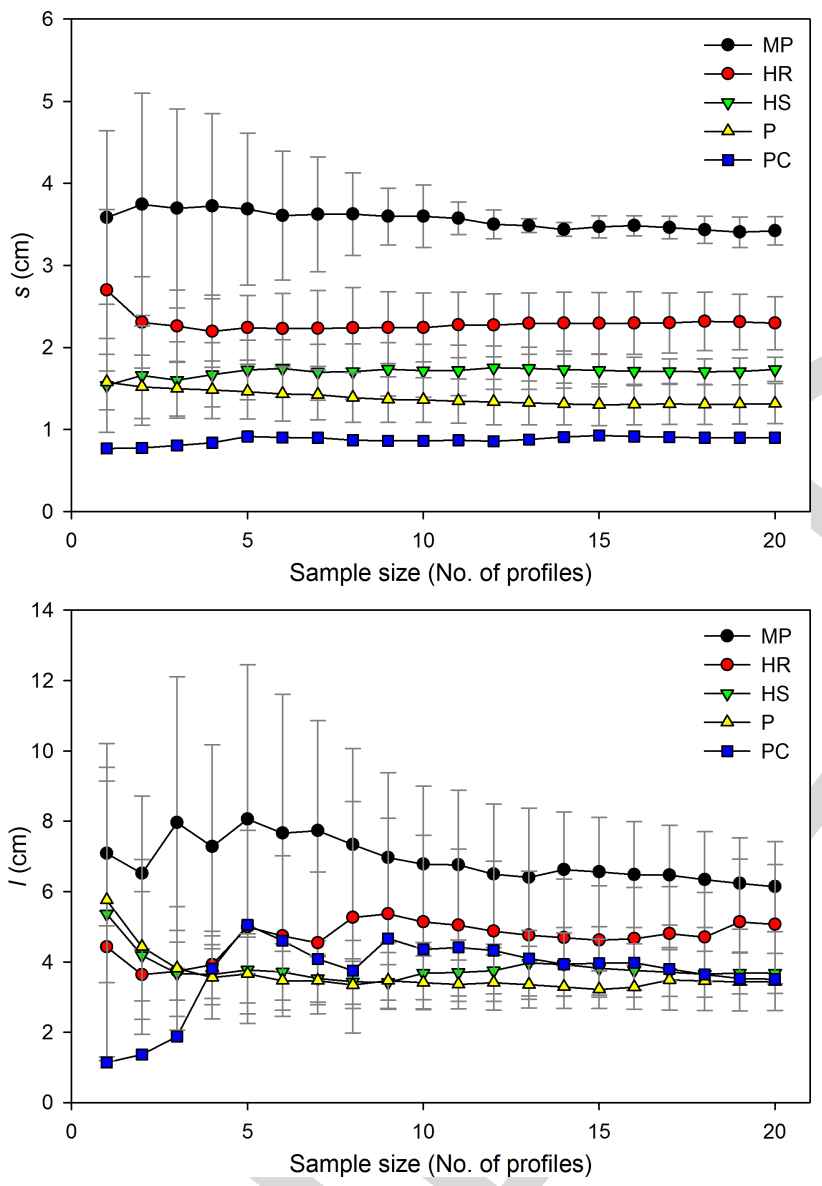

Fig. 1. Mean values of (Top) $s$ and (Bottom) $l$ and their standard deviation (error bars) for different roughness classes depending on the sample size. and rough classes (MP, HR, and HS), respectively, and the semiempirical Oh model [14] that was applicable to all classes.

\section{RESULTS}

\section{A. Behavior of Roughness Parameters}

Mean $s$ values did not change significantly for increasing sample sizes, except for some minor variations when only 1-4 profiles were used (Fig. 1). However, class variability

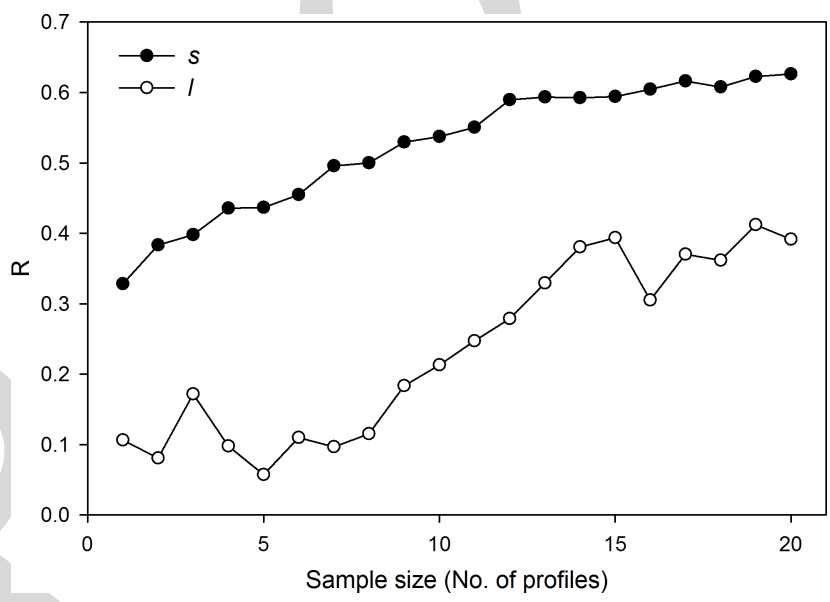

Fig. 2. Spearman correlation coefficient $(R)$ between $\sigma_{\text {norm }}^{0}$ and the roughness parameters $s$ and $l$ depending on sample size.

decreased as the sample size increased, stabilizing for a certain sample size that depended on the particular roughness class. The behavior of $l$ was rather different (Fig. 1), with strongly variable mean values for small sample sizes, which stabilized only after ten profiles. In this case, the reduction of class variability with sample size was slower than that in $s$, being still high for the largest sample sizes analyzed.

Increasing sample sizes resulted in more clustered roughness classes in the $s-l$ space and also in an increase in the correlation between $s$ and $l$ (results not shown). With 20 profiles, a correlation of $R=0.640$ was obtained for the linear function $l=1.89+1.29 \mathrm{~s}$, being similar to that found in [6] in comparable conditions.

\section{B. Roughness Correlation With Backscatter}

The correlation of $\sigma_{\text {norm }}^{0}$ with both roughness parameters for all the sample sizes investigated is presented in Fig. 2. Parameter $s$ showed a steady increase of $R$ as sample size increased, reaching values of $\sim 0.6$ when the number of profiles was larger than 12. Parameter $l$ presented a very low correlation with $\sigma_{\text {norm }}^{0}(R \sim 0.1)$ when the sample size was smaller than eight profiles. When the number of profiles ranged between 8 and 15, it showed a constant increase of correlation, and for 

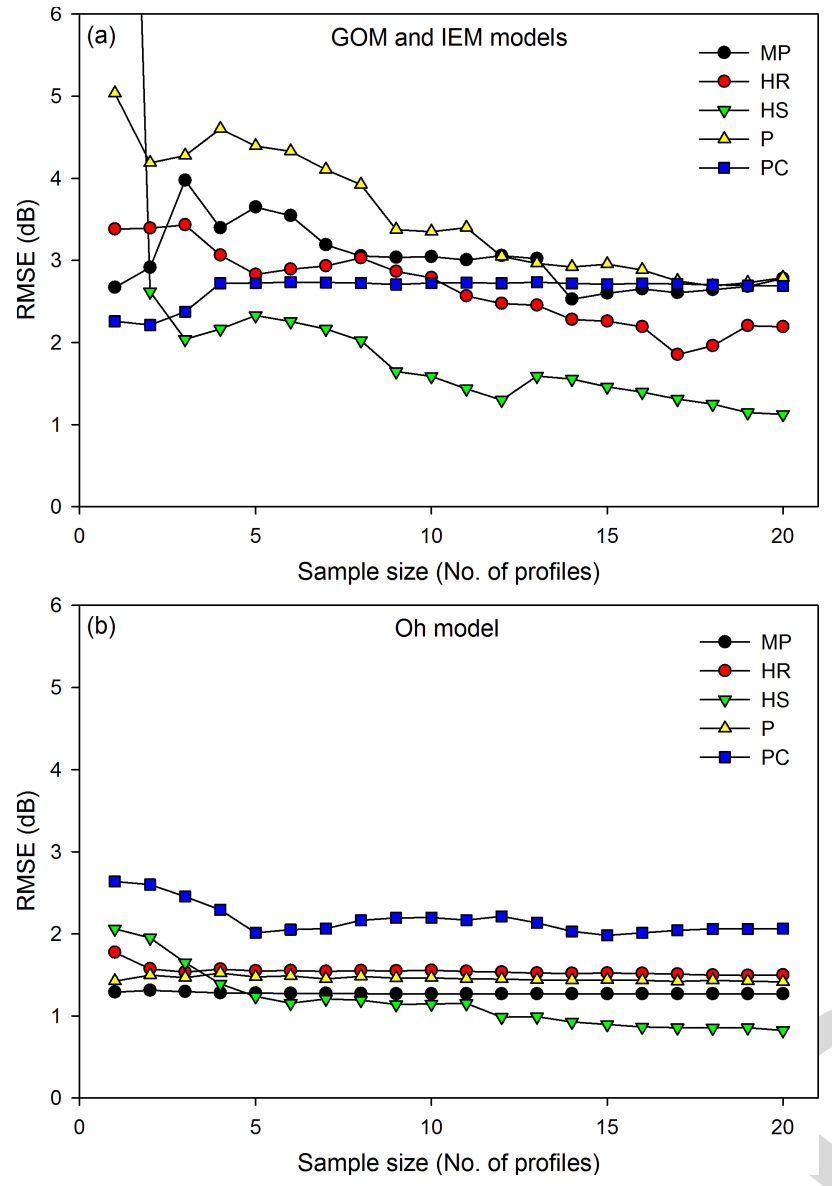

Fig. 3. Roughness class RMSE between simulated and observed field backscatter values depending on sample size. (a) GOM model for classes MP, HR, and HS, and IEM model for classes P and PC. (b) Oh model.

greater sample sizes correlation stabilized at $R \sim 0.4$. Small sample sizes lead to a higher class variability, in particular for $l$ and for the planted (P) roughness class, and this was the main cause for $R$ to drop. When a higher number of samples were used, fields were better clustered around the class mean, leading to higher $R$ values.

\section{Backscatter Modeling}

The goodness of fit of physically based models (IEM and GOM) improved as the sample size increased [Figs. 3(a) and 4(a)-(c)]. The improvements were clear when using the GOM for rough classes (MP, HR, and HS), with RMSE reductions of $\sim 1.5 \mathrm{~dB}$ when passing from 1-5 profiles to 15-20 profiles. Similar RMSE reductions were obtained when applying the IEM to planted fields (P class). In this case, RMSE values passed from $>4 \mathrm{~dB}$ for 1-5 profiles to $\sim 3 \mathrm{~dB}$ for $15-20$ profiles. On the contrary, the class PC had very stable RMSE values $(\sim 2.75 \mathrm{~dB})$, independent of the sample size considered. Considering all the classes, an RMSE of $\sim 2.5 \mathrm{~dB}$ was obtained in the best case [Fig. 4(c)], with the largest residuals corresponding to class $\mathrm{P}$. The best RMSE values achieved per class [Fig. 3(a)] were still high, with values of $2-2.75 \mathrm{~dB}$, except for class $\mathrm{HS}$ with $\sim 1 \mathrm{~dB}$. These values are too high for a viable retrieval of SM from SAR observations.
The semiempirical Oh model also showed a mostly decreasing RMSE trend for increasing sample sizes [Figs. 3(b) and 4(d)-(f)]. However, this decreasing trend was much weaker [Fig. 4(d)-(f)] with an overall RMSE reduction of only $0.078 \mathrm{~dB}$ when passing from 5 to 20 samples. The decreasing trend was different for each of the classes [Fig. 3(b)]. For MP, HR, and P, the RMSE values $(1.2-1.5 \mathrm{~dB})$ were very stable and almost independent of the sample size. Conversely, decreasing RMSE values were observed for HS and PC with some stabilization for sample sizes above five profiles for PC $(\sim 2 \mathrm{~dB})$ and 12 profiles for HS $(\sim 1 \mathrm{~dB})$. The Oh model achieved significantly lower RMSE values than did the GOM and IEM, with largest residuals $(\sim 1-2 \mathrm{~dB})$ obtained at both the lowest and highest ends [Fig. 4(f)], where $\sigma^{0}$ values were underestimated for some rough and smooth fields, respectively. From the analysis, the Oh model seemed to be less sensitive to different sample sizes.

\section{DisCUSSION AND CONCLUSION}

The results obtained illustrate a different behavior of the two classical roughness parameters $s$ and $l$ (Fig. 1). On the one hand, $s$ was rather insensitive to the influence of sample size, with quite stable class means, although, as expected, its variability decreased as the sample size increased. A minimum of 10-15 profiles can be considered sufficient for an accurate determination of $s$. On the other hand, class-mean $l$ values varied more strongly for low sample sizes, and even if its variability decreased for increasing sample sizes, it was still much higher than that of $s$. In this case, depending on the particular roughness class, a sample of 20 profiles might still be insufficient for estimating $l$ with the required accuracy. Similarly, Baghdadi et al. [9] found that averaging ten profiles ( $1 \mathrm{~m}$ long) resulted in quite accurate $s$ estimates $(\sim 10 \%$ error) but much more variable $l$ estimates ( $\sim 20 \%$ error). For larger sample sizes, a significant correlation between $s$ and $l$ was observed, similar to [6]. The existence of an $l=f(s)$ dependence could be used to reduce the number of unknown roughness parameters, which can be important for ill-posed algorithm inversion problems.

The correlation analysis (Fig. 2) revealed a clear sensitivity of backscatter to surface roughness, and in particular $s$, similar to [15]. However, when the number of profiles was insufficient for accurately determining the field mean roughness parameters, $R$ values dropped significantly. On the contrary, for sample sizes $>15$ profiles, $R$ values were as high as 0.6 for $s$ and $\sim 0.35$ for $l$. As the number of samples increased, class variability decreased, leading to better clustered field means that positively correlated with backscatter.

Similar results were obtained when applying the backscatter models (Figs. 3 and 4), with enhanced model precision for larger sample sizes. However, this analysis highlighted the influence of $l$ on the physically based IEM and GOM models. IEM and GOM results were poorer than those obtained with the semiempirical Oh model due to the higher uncertainly of $l$. This could be explained by the larger number of samples required for an accurate estimation of $l$, which caused larger errors in IEM and GOM simulations for a given number of profiles than that in the Oh model. 

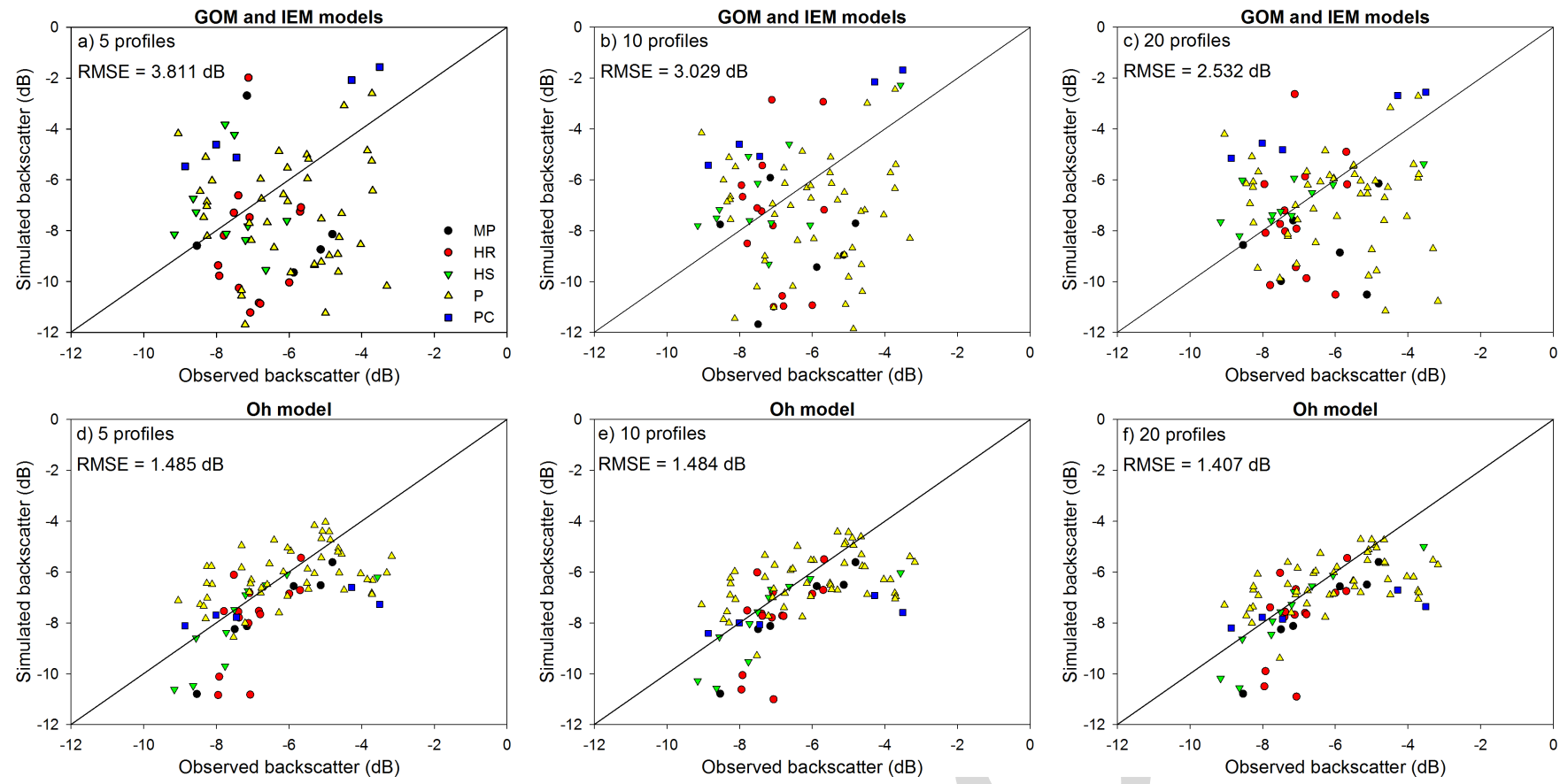

Fig. 4. Goodness of fit between simulated and observed backscatter coefficients per field for different roughness sample sizes with (a)-(c) GOM model for classes MP, HR, and HS and IEM model for classes P and PC, and (d)-(f) Oh model.

To conclude, the results obtained evidence the existing relation between C-band SAR backscatter and SSR for roughness scales shorter than $1 \mathrm{~m}$, as long as a sufficient number of samples are used to accurately characterize roughness. Due to the large spatial variability of roughness parameters, a minimum of ten samples were required for $s$ and a value even larger than 20 might be required for $l$. The lower variability of $s$ caused a better fit of the semiempirical Oh model than that of the physically based IEM and GOM, which were affected by the higher variability of $l$. Altogether, the relatively small errors obtained with the Oh model (between 1 and $1.5 \mathrm{~dB}$ in most cases) recommend its use for the retrieval of SM as long as a minimum of 10-151-m-long roughness profiles are available per field.

\section{ACKNOWLEDGMENT}

H. Lievens is a Post-Doctoral Research Fellow of the Research Foundation Flanders (FWO). The authors would like to thank V. R. N. Pauwels for his collaboration for modeling field soil moisture values with TOPLATS.

\section{REFERENCES}

[1] N. E. C. Verhoest, H. Lievens, W. Wagner, J. Álvarez-Mozos, M. S. Moran, and F. Mattia, "On the soil roughness parameterization problem in soil moisture retrieval of bare surfaces from synthetic aperture radar," Sensors, vol. 8, no. 7, pp. 4213-4248, 2008.

[2] F. T. Ulaby, R. K. Moore, and A. K. Fung, Microwave Remote Sensing: Active and Passive: Radar Remote Sensing and Surface Scattering and Emission Theory (Microwave Remote Sensing), vol. 2. Reading, MA, USA: Addison-Wesley, 1982.

[3] F. Mattia et al., "A comparison between soil roughness statistics used in surface scattering models derived from mechanical and laser profilers,' IEEE Trans. Geosci. Remote Sens., vol. 41, no. 7, pp. 1659-1671, Jul. 2003 .
[4] A. K. Fung, Microwave Scattering and Emission Models and Applications. Norwood, MA, USA: Artech House, 1994.

[5] A. Martinez-Agirre, J. Álvarez-Mozos, H. Lievens, and N. E. C. Verhoest, "Influence of surface roughness measurement scale on radar backscattering in different agricultural soils," IEEE Trans. Geosci. Remote Sens., vol. 55, no. 10, pp. 5925-5936, Oct. 2017, doi: 10.1109/TGRS.2017.2717043.

[6] M. W. J. Davidson et al., "Joint statistical properties of RMS height and correlation length derived from multisite 1-m roughness measurements,' IEEE Trans. Geosci. Remote Sens., vol. 41, no. 7, pp. 1651-1658, Jul. 2003.

[7] N. Baghdadi, N. Holah, and M. Zribi, "Calibration of the integral equation model for SAR data in C-band and $\mathrm{HH}$ and VV polarizations,' Int. J. Remote Sens., vol. 27, no. 4, pp. 805-816, 2006.

[8] R. Bryant et al., "Measuring surface roughness height to parameterize radar backscatter models for retrieval of surface soil moisture," IEEE Geosci. Remote Sens. Lett., vol. 4, no. 1, pp. 137-141, Jan. 2007.

[9] N. Baghdadi et al., "Operational performance of current synthetic aperture radar sensors in mapping soil surface characteristics in agricultural environments: Application to hydrological and erosion modelling," Hydrol. Process., vol. 22, no. 1, pp. 9-20, 2008.

[10] J. Casalí et al., "Runoff, erosion, and water quality of agricultural watersheds in central Navarre (Spain)," Agricult. Water Manage., vol. 95, no. 10, pp. 1111-1128, 2008.

[11] A. Martinez-Agirre, J. Álvarez-Mozos, and R. Giménez, "Evaluation of surface roughness parameters in agricultural soils with different tillage conditions using a laser profile meter," Soil Tillage Res., vol. 161, pp. 19-30, Aug. 2016.

[12] J. S. Famiglietti and E. F. Wood, "Multiscale modeling of spatially variable water and energy balance processes," Water Resour. Res. vol. 30, no. 11, pp. 3061-3078, 1994.

[13] A. K. Fung, Z. Li, and K. S. Chen, "Backscattering from a randomly rough dielectric surface," IEEE Trans. Geosci. Remote Sens., vol. 30, no. 2, pp. 356-369, Mar. 1992.

[14] Y. Oh, K. Sarabandi, and F. T. Ulaby, "An empirical model and an inversion technique for radar scattering from bare soil surfaces," IEEE Trans. Geosci. Remote Sens., vol. 30, no. 2, pp. 370-381, Mar. 1992.

[15] M. Zribi, M. Sahnoun, N. Baghdadi, T. Le Toan, and A. Ben Hamida, "Analysis of the relationship between backscattered P-band radar signals and soil roughness," Remote Sens. Environ., vol. 186, no. 1, pp. 13-21, 2016. 


\section{AUTHOR QUERIES}

\section{AUTHOR PLEASE ANSWER ALL QUERIES}

PLEASE NOTE: We cannot accept new source files as corrections for your paper. If possible, please annotate the PDF proof we have sent you with your corrections and upload it via the Author Gateway. Alternatively, you may send us your corrections in list format. You may also upload revised graphics via the Author Gateway.

Please be aware that authors are required to pay overlength page charges ( $\$ 230$ per page) if the paper is longer than 3 pages. If you cannot pay any or all of these charges please let us know. GRS Society member receive a discounted rate of $\$ 200$ per page.

This pdf contains 2 proofs. The first half is the version that will appear on Xplore. The second half is the version that will appear in print. If you have any figures to print in color, they will be in color in both proofs.

The "Open Access" option for your paper expires when the paper is published on Xplore in an issue with page numbers. Papers in "Early Access" may be changed to Open Access.

If you have not completed your electronic copyright form (ECF) and payment option please return to Scholar One "Transfer Center." In the Transfer Center you will click on "Manuscripts with Decisions" link. You will see your article details and under the "Actions" column click "Transfer Copyright." From the ECF it will direct you to the payment portal to select your payment options and then return to ECF for copyright submission.

$\mathrm{AQ}: 1$ = Please confirm/give details of funding source.

AQ:2 = Please confirm whether the edited sentence "The Spearman R. ." retains the intended meaning.

AQ:3 = Please confirm whether the edited caption for Fig. 4 retains the intended meaning.

AQ:4 = Please confirm whether the retention of the sentence "Hans Lievens is a. .." in the Acknowledgment section is $\mathrm{OK}$. 


\title{
Influence of Surface Roughness Sample Size for C-Band SAR Backscatter Applications on Agricultural Soils
}

\author{
Alex Martinez-Agirre ${ }^{\mathbb{D}}$, Jesús Álvarez-Mozos, Hans Lievens, Niko E. C. Verhoest, and Rafael Giménez
}

\begin{abstract}
Soil surface roughness determines the backscatter coefficient observed by radar sensors. The objective of this letter was to determine the surface roughness sample size required in synthetic aperture radar applications and to provide some guidelines on roughness characterization in agricultural soils for these applications. With this aim, a data set consisting of ten ENVISAT/ASAR observations acquired coinciding with soil moisture and surface roughness surveys has been processed. The analysis consisted of: 1) assessing the accuracies of roughness parameters $s$ and $l$ depending on the number of 1 -m-long profiles measured per field; 2) computing the correlation of field average roughness parameters with backscatter observations; and 3) evaluating the goodness of fit of three widely used backscatter models, i.e., integral equation model (IEM), geometrical optics model (GOM), and Oh model. The results obtained illustrate a different behavior of the two roughness parameters. A minimum of 10-15 profiles can be considered sufficient for an accurate determination of $s$, while 20 profiles might still be not enough for accurately estimating $l$. The correlation analysis revealed a clear sensitivity of backscatter to surface roughness. For sample sizes $>15$ profiles, $R$ values were as high as 0.6 for $s$ and $\sim 0.35$ for $l$, while for smaller sample sizes $R$ values dropped significantly. Similar results were obtained when applying the backscatter models, with enhanced model precision for larger sample sizes. However, IEM and GOM results were poorer than those obtained with the Oh model and more affected by lower sample sizes, probably due to larger uncertainly of $l$.
\end{abstract}

Index Terms-Agricultural soils, backscatter models, surface roughness, synthetic aperture radar (SAR).

\section{INTRODUCTION}

S YNTHETIC aperture radar (SAR) sensors measure the backscatter of observed targets and offer valuable information for the identification of terrain covers and for the

Manuscript received August 31, 2017; accepted October 8, 2017. This work was supported by the Spanish Ministry of Economy and Competitiveness through scholarship under Grant BES-2012-054521 and through MINECO/FEDER, EU, under Project CGL2011-24336, Project CGL201564284-C2-1-R, and Project CGL2016-75217-R. (Corresponding author: Alex Martinez-Agirre.)

A. Martinez-Agirre, J. Álvarez-Mozos, and R. Giménez are with the Department of Projects and Rural Engineering, IS-FOOD Institute (Innovation and Sustainable Development in Food Chain), Public University of Navarre, 31006 Pamplona, Spain (e-mail: alejandro.mda@unavarra.es).

H. Lievens is with the Laboratory of Hidrology and Water Management, Ghent University, B-9000 Ghent, Belgium, and also with the Global Modeling and Assimilation Office, NASA Goddard Space Flight Center, Greenbelt, MD 20771 USA.

N. E. C. Verhoest is with the Laboratory of Hidrology and Water Management, Ghent University, B-9000 Ghent, Belgium.

Color versions of one or more of the figures in this letter are available online at http://ieeexplore.ieee.org.

Digital Object Identifier 10.1109/LGRS.2017.2762434 retrieval of biogeophysical parameters of interest, such as soil moisture (SM), vegetation phenology, and biomass. Among other terrain parameters, soil surface roughness (SSR) strongly affects the scattering of microwaves, and hence largely determines the backscatter coefficient $\left(\sigma^{0}\right)$ observed by radar sensors, complicating the interpretation and analysis of SAR data [1]. In the SAR literature, SSR has been mostly parameterized by the standard deviation of the heights $(s)$, the correlation length $(l)$, and the shape of the autocorrelation function [2], generally assumed exponential for agricultural soils. Several backscatter models exist that use these parameters as input for simulating $\sigma^{0}$. If backscatter observations are available, models can be inverted for retrieving a certain terrain parameter of interest (normally SM). An accurate estimation of roughness parameters is a prerequisite for this. Yet, their spatial variability and also the multiscalar nature of roughness make it difficult to determine $s$ and $l$ values with the required accuracy for obtaining useful inversions [2].

Surface roughness is known to be a multiscalar phenomenon, causing instruments with different measuring ranges (i.e., profile length or surveying area) yield parameter values that are not comparable with each other [2]. In particular, the presence of long-wavelength roughness components (i.e., several meters) on a soil surface or profile can strongly affect the shape of the obtained autocorrelation functions, introducing uncertainty in the determination of $l$ [3]. On the other hand, recent research has evidenced that these long-wavelength components might not play a significant role in the scattering of microwaves at the frequencies used by earth observation satellites [4], [5]. This is in line with previous studies that used profile lengths of 1-2 $\mathrm{m}$ for surface roughness characterization with good results [6], [7].

However, due to the spatial variability of surface roughness, a minimum amount of samples might be required for accurately characterizing roughness parameters for a particular agricultural field or roughness class. Bryant et al. [8] observed that at least 20 profiles were required to accurately determine $s$. Similarly, Baghdadi et al. [9] reported a $\pm 10 \%$ accuracy for parameter $s$ and $\pm 20 \%$ for $l$ when ten roughness profiles were used. Yet, it is necessary not only to assess how the roughness sample size (i.e., number of profiles measured) affects the accuracy of the computed parameters, but also to evaluate how it influences the accuracy of backscatter simulations using observed $\sigma^{0}$ data.

The aim of this letter was to evaluate the influence of surface roughness sample size on SAR backscattering in different 
TABLE I

Roughness Classes CorResponding to EACH Field AND Measurement Date. Four 5-m-Long Roughness PROFILES WERE ACQUIRED PER FIELD

\begin{tabular}{ccccc}
\hline Field ID. & $\mathbf{2 2 / 0 9 / 2 0 0 4}$ & $\mathbf{0 8 / 1 0 / 2 0 0 4}$ & $\mathbf{2 4 / 1 0 / 2 0 0 4}$ & $\mathbf{1 7 / 1 2 / 2 0 0 4}$ \\
\hline 188 & HR & HR & - & $\mathrm{P}$ \\
189 & HR & HR & HS & P \\
193 & HR & HR & P & P \\
194 & - & HR & HR & P \\
199 & MP & MP & MP & P \\
201 & HS & HS & - & P \\
208 & MP & - & - & PC \\
235 & HS & HS & P & P \\
255 & HS & HS & - & P \\
258 & HR & - & - & P \\
\hline
\end{tabular}

- Fields not monitored on that particular day

agricultural soils. The objective was to determine the minimum number of 1-m-long profiles required in SAR applications and to provide same guidelines on how roughness should be characterized for these applications. With this aim, a data set consisting of ENVISAT/ASAR observations acquired coinciding with some ground surveys has been processed. The analysis consisted of: 1) assessing the accuracies of $s$ and $l$ depending on the number of profiles measured per field; 2) computing the correlation of field average roughness parameters with backscatter; and 3) evaluating the goodness of fit of backscatter models depending on the roughness sample size considered.

\section{Materials And Methods}

\section{A. Test Site}

The experimental data acquisition was carried out on the watershed of La Tejería (N42 $44^{\prime} 10.6^{\prime \prime}$ and W1 $\left.{ }^{\circ} 56^{\prime} 57.2^{\prime \prime}\right)$ in Navarre (Spain) [10]. The climate is humid sub-Mediterranean with a mean annual temperature of $13{ }^{\circ} \mathrm{C}$ and an average annual precipitation of $\sim 700 \mathrm{~mm}$. Soils have a silty-clay texture and are relatively shallow (0.5-1 m deep). Ten agricultural fields were studied with an area ranging between 3 and 7.3 ha.

Soil preparation operations were performed sequentially during September and October 2004 for cultivating winter cereal. Five different tillage treatments were observed from September to December 2004 (Table I): mouldboard plough (MP), harrowed rough (HR), harrowed smooth (HS), planted (P), and planted compacted (PC).

\section{B. Surface Roughness Data}

Surface roughness was measured using a 5-m-long laser profile meter with a resolution of $5 \mathrm{~mm}$ and a vertical accuracy of $1.25 \mathrm{~mm}$ [11]. On each monitored field, four 5-m-long profiles were measured per date (except for field 208 on September 22, 2004), spatially distributed throughout the field and in parallel to the tillage direction. Each acquired profile was subdivided into five 1-m-long profiles, and these were detrended using a linear function to subtract the terrain slope. Thus, 20 1-m-long profiles (i.e., independent samples) were obtained per field, making a total of 6351 -m-long profiles.
Two standard surface roughness parameters were analyzed: the standard deviation of surface heights $(s)$ and the correlation length $(l)$ obtained considering an exponential autocorrelation function [2]. Further details on the processing of profiles and roughness parameters are available in [11].

\section{Soil Moisture Data}

SM was measured using a commercial time domain reflectometry (TDR) instrument. On each field, five spatially distributed measurement locations were monitored per date. Soil samples were used to calibrate the TDR probe. Also, TOPLATS [12]-modeled SM values were used for four satellite acquisition dates (Table II) on which the TDR measurements were not available.

\section{SAR Data}

Ten ENVISAT/ASAR scenes were acquired over La Tejería watershed during the study period (Table II). Scenes were acquired as VV single-pol image mode precision image products in swath IS2 (except for September 22, 2004, that was VV/HH Alternate Pol in IS1), half of them in ascending pass and the other half in descending. In all cases, the resolution was $30 \mathrm{~m} \times 30 \mathrm{~m}$. Scenes were: 1) orthorectified (with an error $<1$ pixel); 2) calibrated (using the local incidence angle); and 3 ) speckle filtered (gamma MAP filter with a window of $5 \times 5$ ). Mean backscatter coefficient values $\sigma^{0}$ were calculated for each field per date.

\section{E. Data Analysis}

The analysis presented here focused on the influence of sample size on the characterization of surface roughness for SAR applications. For this, an increasing number of 1-m-long roughness profiles (from 1 to 20) were considered for each field, and the following analyses were carried out: 1) assessment of the behavior of roughness parameters; 2) evaluation of the correlation between normalized $\sigma^{0}$ and roughness parameters; and 3) evaluation of the goodness of fit of different backscatter models.

The behavior of roughness parameters was evaluated by comparing the average and standard deviation of $s$ and $l$ per class computed considering an increasing sample size (i.e., number of profiles). For the correlation analysis, field average $\sigma^{0}$ values were normalized for incidence angle and SM variations, so as to remove the influence of factors other than surface roughness on $\sigma^{0}$ values [5]. Further details on the normalization can be found in [5]. The Spearman coefficient $(R)$ was computed between the $\sigma_{\text {norm }}^{0}$ (normalized $\sigma^{0}$ ) series and field average $s$ and $l$ values considering an increasing sample size. Finally, the goodness of fit of three backscatter models was evaluated by computing the root-mean-square error (RMSE) between observed $\sigma^{0}$ values and simulated ones; the latter were obtained using field average $s$ and $l$ values for an increasing sample size. Due to their different nature and validity range, three backscatter models were considered: the physically based integral equation model (IEM) [13] and geometrical optics model (GOM) [2] for the smooth (P and PC) 
TABLE II

SUMMARY OF SAR DATA

\begin{tabular}{clccccc}
\hline Date & SAR data & $\boldsymbol{\theta}_{\text {Loc }}\left(\mathbf{(}^{*}\right.$ & Pass & No. Fields & Roughness data & SM data \\
\hline $22 / 09 / 2004$ & Envisat/ASAR & $7.2-16.2$ & Descending & 9 & Profilometer & TDR \\
$08 / 10 / 2004$ & Envisat/ASAR & $11.6-20.9$ & Descending & 8 & Profilometer & TDR \\
$11 / 10 / 2004$ & Envisat/ASAR & $20.9-31.4$ & Ascending & 8 & $=$ & TOPLATS \\
$24 / 10 / 2004$ & Envisat/ASAR & $15.7-24.9$ & Descending & 5 & Profilometer & TDR \\
$27 / 10 / 2004$ & Envisat/ASAR & $16.9-27.2$ & Ascending & 5 & $=$ & TOPLATS \\
$17 / 12 / 2004$ & Envisat/ASAR & $11.6-20.9$ & Descending & 10 & Profilometer & TDR \\
$20 / 12 / 2004$ & Envisat/ASAR & $20.8-31.2$ & Ascending & 10 & $=$ & TOPLATS \\
$02 / 01 / 2005$ & Envisat/ASAR & $15.8-24.5$ & Descending & 10 & $=$ & TDR \\
$05 / 01 / 2005$ & Envisat/ASAR & $16.8-26.9$ & Ascending & 10 & $=$ & TDR \\
$24 / 01 / 2005$ & Envisat/ASAR & $20.9-31.3$ & Ascending & 10 & $=$ & TOPLATS \\
\hline
\end{tabular}

*Local incidence angle
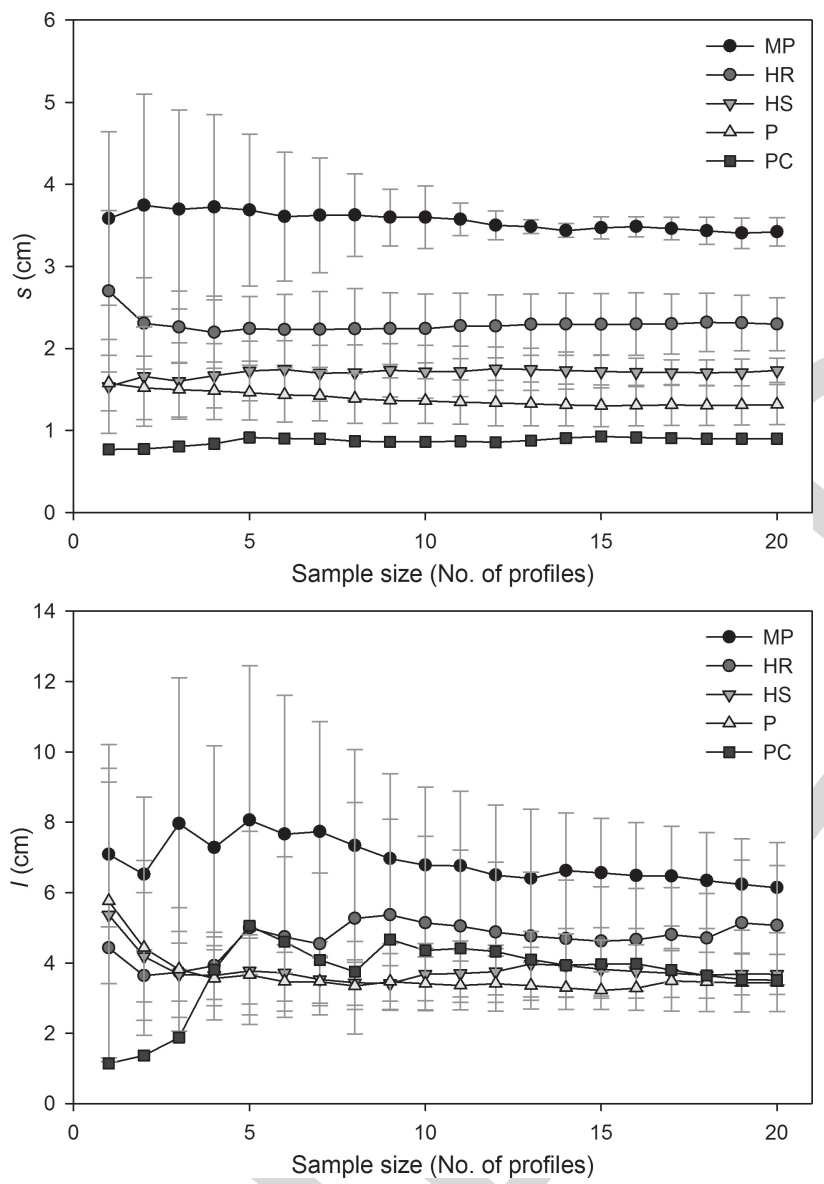

Fig. 1. Mean values of (Top) $s$ and (Bottom) $l$ and their standard deviation (error bars) for different roughness classes depending on the sample size. and rough classes (MP, HR, and HS), respectively, and the semiempirical Oh model [14] that was applicable to all classes.

\section{RESULTS}

\section{A. Behavior of Roughness Parameters}

Mean $s$ values did not change significantly for increasing sample sizes, except for some minor variations when only 1-4 profiles were used (Fig. 1). However, class variability

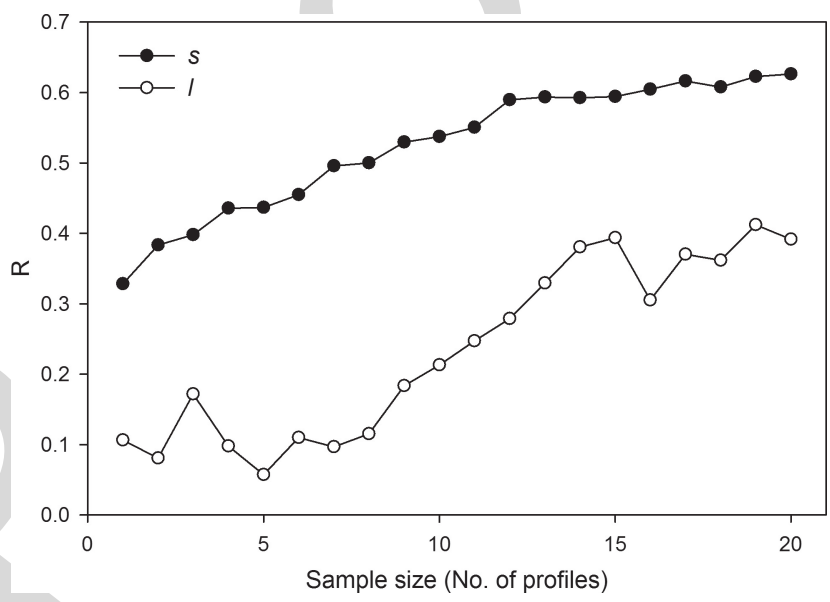

Fig. 2. Spearman correlation coefficient $(R)$ between $\sigma_{\text {norm }}^{0}$ and the roughness parameters $s$ and $l$ depending on sample size.

decreased as the sample size increased, stabilizing for a certain sample size that depended on the particular roughness class. The behavior of $l$ was rather different (Fig. 1), with strongly variable mean values for small sample sizes, which stabilized only after ten profiles. In this case, the reduction of class variability with sample size was slower than that in $s$, being still high for the largest sample sizes analyzed.

Increasing sample sizes resulted in more clustered roughness classes in the $s-l$ space and also in an increase in the correlation between $s$ and $l$ (results not shown). With 20 profiles, a correlation of $R=0.640$ was obtained for the linear function $l=1.89+1.29 \mathrm{~s}$, being similar to that found in [6] in comparable conditions.

\section{B. Roughness Correlation With Backscatter}

The correlation of $\sigma_{\text {norm }}^{0}$ with both roughness parameters for all the sample sizes investigated is presented in Fig. 2. Parameter $s$ showed a steady increase of $R$ as sample size increased, reaching values of $\sim 0.6$ when the number of profiles was larger than 12. Parameter $l$ presented a very low correlation with $\sigma_{\text {norm }}^{0}(R \sim 0.1)$ when the sample size was smaller than eight profiles. When the number of profiles ranged between 8 and 15, it showed a constant increase of correlation, and for 

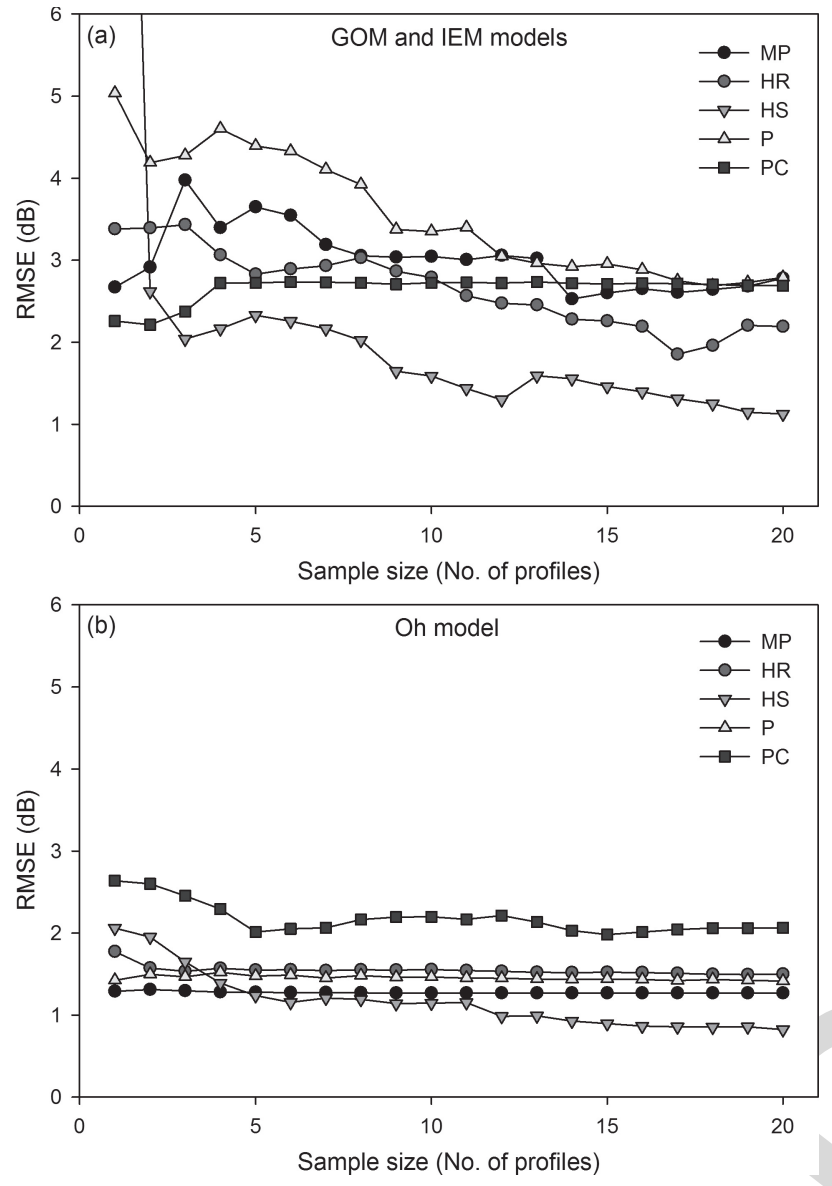

Fig. 3. Roughness class RMSE between simulated and observed field backscatter values depending on sample size. (a) GOM model for classes MP, HR, and HS, and IEM model for classes P and PC. (b) Oh model.

greater sample sizes correlation stabilized at $R \sim 0.4$. Small sample sizes lead to a higher class variability, in particular for $l$ and for the planted (P) roughness class, and this was the main cause for $R$ to drop. When a higher number of samples were used, fields were better clustered around the class mean, leading to higher $R$ values.

\section{Backscatter Modeling}

The goodness of fit of physically based models (IEM and GOM) improved as the sample size increased [Figs. 3(a) and 4(a)-(c)]. The improvements were clear when using the GOM for rough classes (MP, HR, and HS), with RMSE reductions of $\sim 1.5 \mathrm{~dB}$ when passing from 1-5 profiles to 15-20 profiles. Similar RMSE reductions were obtained when applying the IEM to planted fields (P class). In this case, RMSE values passed from $>4 \mathrm{~dB}$ for 1-5 profiles to $\sim 3 \mathrm{~dB}$ for $15-20$ profiles. On the contrary, the class PC had very stable RMSE values $(\sim 2.75 \mathrm{~dB})$, independent of the sample size considered. Considering all the classes, an RMSE of $\sim 2.5 \mathrm{~dB}$ was obtained in the best case [Fig. 4(c)], with the largest residuals corresponding to class $\mathrm{P}$. The best RMSE values achieved per class [Fig. 3(a)] were still high, with values of $2-2.75 \mathrm{~dB}$, except for class $\mathrm{HS}$ with $\sim 1 \mathrm{~dB}$. These values are too high for a viable retrieval of SM from SAR observations.
The semiempirical Oh model also showed a mostly decreasing RMSE trend for increasing sample sizes [Figs. 3(b) and 4(d)-(f)]. However, this decreasing trend was much weaker [Fig. 4(d)-(f)] with an overall RMSE reduction of only $0.078 \mathrm{~dB}$ when passing from 5 to 20 samples. The decreasing trend was different for each of the classes [Fig. 3(b)]. For MP, HR, and P, the RMSE values $(1.2-1.5 \mathrm{~dB})$ were very stable and almost independent of the sample size. Conversely, decreasing RMSE values were observed for HS and PC with some stabilization for sample sizes above five profiles for PC $(\sim 2 \mathrm{~dB})$ and 12 profiles for HS $(\sim 1 \mathrm{~dB})$. The Oh model achieved significantly lower RMSE values than did the GOM and IEM, with largest residuals $(\sim 1-2 \mathrm{~dB})$ obtained at both the lowest and highest ends [Fig. 4(f)], where $\sigma^{0}$ values were underestimated for some rough and smooth fields, respectively. From the analysis, the Oh model seemed to be less sensitive to different sample sizes.

\section{DisCUSSION AND CONCLUSION}

The results obtained illustrate a different behavior of the two classical roughness parameters $s$ and $l$ (Fig. 1). On the one hand, $s$ was rather insensitive to the influence of sample size, with quite stable class means, although, as expected, its variability decreased as the sample size increased. A minimum of 10-15 profiles can be considered sufficient for an accurate determination of $s$. On the other hand, class-mean $l$ values varied more strongly for low sample sizes, and even if its variability decreased for increasing sample sizes, it was still much higher than that of $s$. In this case, depending on the particular roughness class, a sample of 20 profiles might still be insufficient for estimating $l$ with the required accuracy. Similarly, Baghdadi et al. [9] found that averaging ten profiles ( $1 \mathrm{~m}$ long) resulted in quite accurate $s$ estimates $(\sim 10 \%$ error) but much more variable $l$ estimates ( $\sim 20 \%$ error). For larger sample sizes, a significant correlation between $s$ and $l$ was observed, similar to [6]. The existence of an $l=f(s)$ dependence could be used to reduce the number of unknown roughness parameters, which can be important for ill-posed algorithm inversion problems.

The correlation analysis (Fig. 2) revealed a clear sensitivity of backscatter to surface roughness, and in particular $s$, similar to [15]. However, when the number of profiles was insufficient for accurately determining the field mean roughness parameters, $R$ values dropped significantly. On the contrary, for sample sizes $>15$ profiles, $R$ values were as high as 0.6 for $s$ and $\sim 0.35$ for $l$. As the number of samples increased, class variability decreased, leading to better clustered field means that positively correlated with backscatter.

Similar results were obtained when applying the backscatter models (Figs. 3 and 4), with enhanced model precision for larger sample sizes. However, this analysis highlighted the influence of $l$ on the physically based IEM and GOM models. IEM and GOM results were poorer than those obtained with the semiempirical Oh model due to the higher uncertainly of $l$. This could be explained by the larger number of samples required for an accurate estimation of $l$, which caused larger errors in IEM and GOM simulations for a given number of profiles than that in the Oh model. 

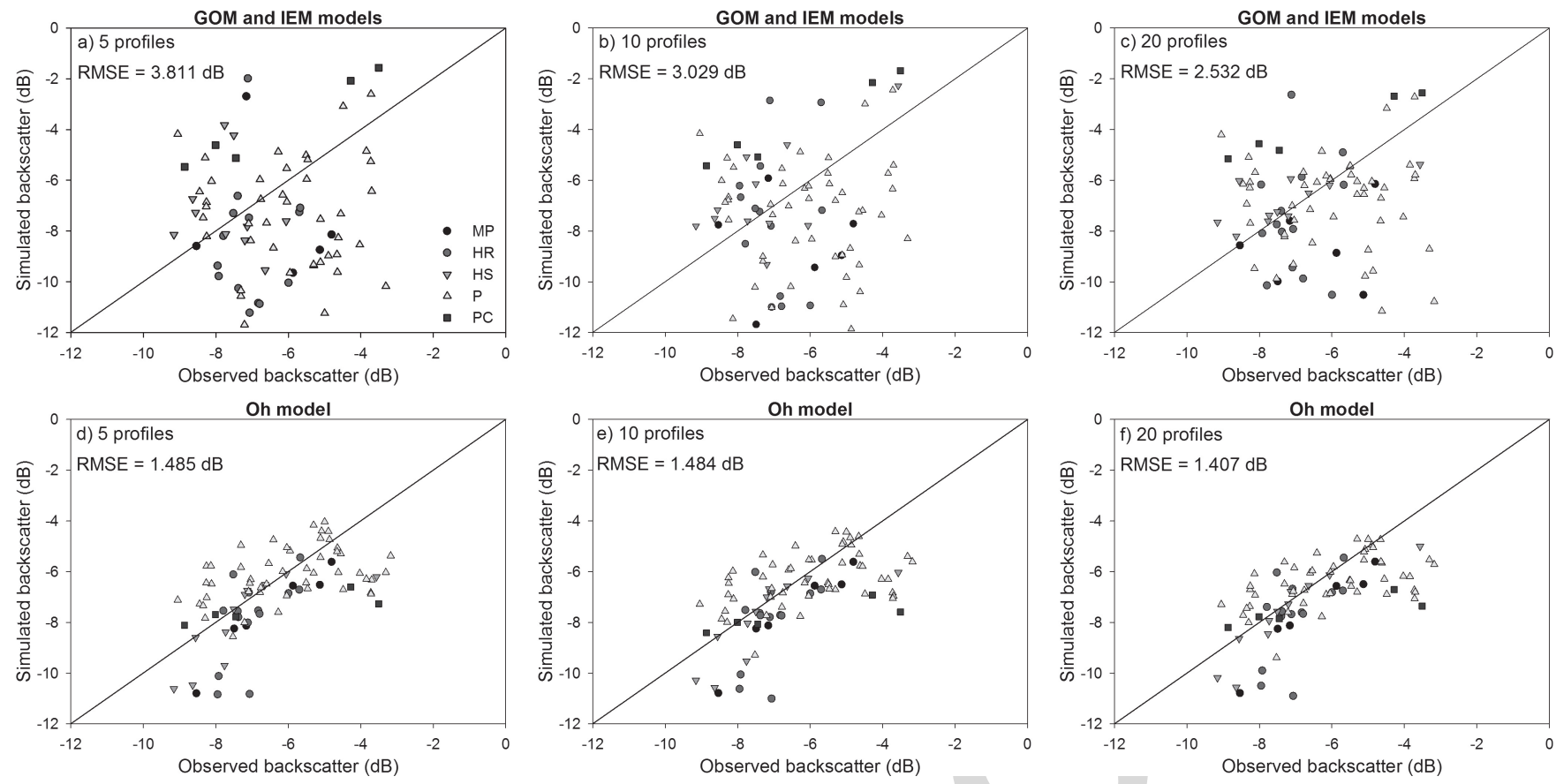

Fig. 4. Goodness of fit between simulated and observed backscatter coefficients per field for different roughness sample sizes with (a)-(c) GOM model for classes MP, HR, and HS and IEM model for classes P and PC, and (d)-(f) Oh model.

To conclude, the results obtained evidence the existing relation between C-band SAR backscatter and SSR for roughness scales shorter than $1 \mathrm{~m}$, as long as a sufficient number of samples are used to accurately characterize roughness. Due to the large spatial variability of roughness parameters, a minimum of ten samples were required for $s$ and a value even larger than 20 might be required for $l$. The lower variability of $s$ caused a better fit of the semiempirical Oh model than that of the physically based IEM and GOM, which were affected by the higher variability of $l$. Altogether, the relatively small errors obtained with the Oh model (between 1 and $1.5 \mathrm{~dB}$ in most cases) recommend its use for the retrieval of SM as long as a minimum of 10-15 1-m-long roughness profiles are available per field.

\section{ACKNOWLEDGMENT}

H. Lievens is a Post-Doctoral Research Fellow of the Research Foundation Flanders (FWO). The authors would like to thank V. R. N. Pauwels for his collaboration for modeling field soil moisture values with TOPLATS.

\section{REFERENCES}

[1] N. E. C. Verhoest, H. Lievens, W. Wagner, J. Álvarez-Mozos, M. S. Moran, and F. Mattia, "On the soil roughness parameterization problem in soil moisture retrieval of bare surfaces from synthetic aperture radar," Sensors, vol. 8, no. 7, pp. 4213-4248, 2008.

[2] F. T. Ulaby, R. K. Moore, and A. K. Fung, Microwave Remote Sensing: Active and Passive: Radar Remote Sensing and Surface Scattering and Emission Theory (Microwave Remote Sensing), vol. 2. Reading, MA, USA: Addison-Wesley, 1982.

[3] F. Mattia et al., "A comparison between soil roughness statistics used in surface scattering models derived from mechanical and laser profilers,' IEEE Trans. Geosci. Remote Sens., vol. 41, no. 7, pp. 1659-1671, Jul. 2003.
[4] A. K. Fung, Microwave Scattering and Emission Models and Applications. Norwood, MA, USA: Artech House, 1994.

[5] A. Martinez-Agirre, J. Álvarez-Mozos, H. Lievens, and N. E. C. Verhoest, "Influence of surface roughness measurement scale on radar backscattering in different agricultural soils," IEEE Trans. Geosci. Remote Sens., vol. 55, no. 10, pp. 5925-5936, Oct. 2017, doi: 10.1109/TGRS.2017.2717043.

[6] M. W. J. Davidson et al., "Joint statistical properties of RMS height and correlation length derived from multisite 1-m roughness measurements,' IEEE Trans. Geosci. Remote Sens., vol. 41, no. 7, pp. 1651-1658, Jul. 2003.

[7] N. Baghdadi, N. Holah, and M. Zribi, "Calibration of the integral equation model for SAR data in C-band and HH and VV polarizations," Int. J. Remote Sens., vol. 27, no. 4, pp. 805-816, 2006.

[8] R. Bryant et al., "Measuring surface roughness height to parameterize radar backscatter models for retrieval of surface soil moisture," IEEE Geosci. Remote Sens. Lett., vol. 4, no. 1, pp. 137-141, Jan. 2007.

[9] N. Baghdadi et al., "Operational performance of current synthetic aperture radar sensors in mapping soil surface characteristics in agricultural environments: Application to hydrological and erosion modelling," Hydrol. Process., vol. 22, no. 1, pp. 9-20, 2008.

[10] J. Casalí et al., "Runoff, erosion, and water quality of agricultural watersheds in central Navarre (Spain)," Agricult. Water Manage., vol. 95, no. 10, pp. 1111-1128, 2008.

[11] A. Martinez-Agirre, J. Álvarez-Mozos, and R. Giménez, "Evaluation of surface roughness parameters in agricultural soils with different tillage conditions using a laser profile meter," Soil Tillage Res., vol. 161, pp. 19-30, Aug. 2016.

[12] J. S. Famiglietti and E. F. Wood, "Multiscale modeling of spatially variable water and energy balance processes," Water Resour. Res. vol. 30, no. 11, pp. 3061-3078, 1994.

[13] A. K. Fung, Z. Li, and K. S. Chen, "Backscattering from a randomly rough dielectric surface," IEEE Trans. Geosci. Remote Sens., vol. 30 no. 2, pp. 356-369, Mar. 1992.

[14] Y. Oh, K. Sarabandi, and F. T. Ulaby, "An empirical model and an inversion technique for radar scattering from bare soil surfaces," IEEE Trans. Geosci. Remote Sens., vol. 30, no. 2, pp. 370-381, Mar. 1992.

[15] M. Zribi, M. Sahnoun, N. Baghdadi, T. Le Toan, and A. Ben Hamida, "Analysis of the relationship between backscattered P-band radar signals and soil roughness," Remote Sens. Environ., vol. 186, no. 1, pp. 13-21, 2016. 


\section{AUTHOR QUERIES}

\section{AUTHOR PLEASE ANSWER ALL QUERIES}

PLEASE NOTE: We cannot accept new source files as corrections for your paper. If possible, please annotate the PDF proof we have sent you with your corrections and upload it via the Author Gateway. Alternatively, you may send us your corrections in list format. You may also upload revised graphics via the Author Gateway.

Please be aware that authors are required to pay overlength page charges ( $\$ 230$ per page) if the paper is longer than 3 pages. If you cannot pay any or all of these charges please let us know. GRS Society member receive a discounted rate of $\$ 200$ per page.

This pdf contains 2 proofs. The first half is the version that will appear on Xplore. The second half is the version that will appear in print. If you have any figures to print in color, they will be in color in both proofs.

The "Open Access" option for your paper expires when the paper is published on Xplore in an issue with page numbers. Papers in "Early Access" may be changed to Open Access.

If you have not completed your electronic copyright form (ECF) and payment option please return to Scholar One "Transfer Center." In the Transfer Center you will click on "Manuscripts with Decisions" link. You will see your article details and under the "Actions" column click "Transfer Copyright." From the ECF it will direct you to the payment portal to select your payment options and then return to ECF for copyright submission.

$\mathrm{AQ}: 1$ = Please confirm/give details of funding source.

AQ:2 = Please confirm whether the edited sentence "The Spearman R. ." retains the intended meaning.

AQ:3 = Please confirm whether the edited caption for Fig. 4 retains the intended meaning.

AQ:4 = Please confirm whether the retention of the sentence "Hans Lievens is a. .." in the Acknowledgment section is $\mathrm{OK}$. 